\title{
A Study on Subchronic Inhalation Toxicity of 1-Chloropropane
}

\author{
Yong Hyun Chung, Jeong Hee Han and Yong-Hoon Lee \\ Toxicity Research Team, Occupational Safety and Health Research Institute, KOSHA, Daejeon, Korea
}

(Received July 23, 2015; Revised September 11, 2015; Accepted November 12, 2015)

\begin{abstract}
This study was conducted to measure toxicity of 1 -chloropropane (CAS No. : 540-54-5). According to the OECD Test Guideline 413 (Subchronic inhalation toxicity: 90-day study), SD rats were exposed to 0, 310, 1,250 , and 5,000 ppm of 1-chloropropane for $6 \mathrm{~h} /$ day, 5 day/week for 13 weeks via whole-body inhalation. Mortality, clinical signs, body weights, food consumption, motor activity, ophthalmoscopy, hematology, serum chemistry, urinalysis, organ weights, gross and histopathological findings were compared between control and all tested groups. No mortality or remarkable clinical signs were examined during the study. No gross lesions or adverse effects on body weight, food consumption, motor activity, ophthalmoscopy, urinalysis, hematology, organ weights were observed in any of male or female rats in all tested groups. In serum biochemistry, glucose was significantly decreased in males of 1,250 and 5,000 ppm groups compared to control group in dose-dependent relationship. In histopathological examination, vacuolation of acinar cells was observed in pancreas of all male and female groups exposed to 1 -chloropropane. In conclusion, no observable adverse effect level (NOAEL) was considered to be below $310 \mathrm{ppm} /$ $6 \mathrm{~h} /$ day, 5 day/week for rats.
\end{abstract}

Key words: 1-Chloropropane, NOAEL, Subchronic inhalation toxicity

\section{INTRODUCTION}

1-Chloropropane (CAS No. 540-54-5) is a flammable, colorless liquid with chloroform odor and is prepared by reacting $n$-propyl alcohol with hydrochloric acid in the presence of a zinc chloride as a catalyst (1). It is an organic compound with chemical formula $\mathrm{CH}_{3} \mathrm{CH}_{2} \mathrm{CH}_{2} \mathrm{Cl}$ and vapor pressure of $345 \mathrm{~mm} \mathrm{Hg}$ at $25^{\circ} \mathrm{C}$. 1-chloropropane is used as a chemical intermediate for $n$-Propylamine and has been studied as an anesthetic and parasiticidal drug $(1,2)$. In addition, it is also used as an intermediate to proude the sweetener sodium 2-(4-methoxybenzoyl) benzoate used for food and beverages, but their applicability had been limited (1). The annual production of 1 -chloropropane in Korea was 249.9 tons in 2006 and is mainly used in domestic region (3).

The toxicity of 1 -chloropropane has been rarely docu-

Correspondence to: Yong-Hoon Lee, Toxicity Research Team, Occupational Safety and Health Research Institute, KOSHA, 339-30 Expo-ro, Yuseong-gu, Daejeon 305-380, Korea

E-mail: dvmone@kosha.or.kr

This is an Open-Access article distributed under the terms of the Creative Commons Attribution Non-Commercial License (http:// creativecommons.org/licenses/by-nc/3.0) which permits unrestricted non-commercial use, distribution, and reproduction in any medium, provided the original work is properly cited. mented in humans and experimental animals. The inhalation exposure of 1-chloropropane in human cause central nervous system effects characterized by nausea, headache, dizziness, unconsciousness, coma and may cause respiratory irritation. Also, liquid form of 1-chloropropane can cause eye, skin, and digestive irritation (4). 1-chloropropane toxicological experiments demonstrated that 1-chloropropane has $\mathrm{LD}_{50}$ value of $>2,000 \mathrm{mg} / \mathrm{kg}$ in rats for acute oral toxicity (5). Also, short term inhalation study of 1-chloropropane revealed that slight alveolar hemorrhage of the lungs and significant focal necrosis of the liver were observed in rats exposed to $40,000 \mathrm{ppm} 1 \mathrm{hr} /$ day for 4 days (6). Eye irritation in rabbits is also reported for 1 -chloropropane (7). 1-chloropropane is volatile and can cause most adverse effects via inhalation. Therefore, workers exposed to it via inhalation have the potential health risk at the workplace. However, there is a lack of information regarding long term repeated-dose toxicity by inhalation.

Therefore, this study was conducted to characterize the potential subchronic inhalation toxicity of 1-chloropropane by whole-body exposure in Sprague-Dawley (SD) rats in accordance with Good Laboratory Practice (GLP) guideline and the test guideline No. 413 "Subchronic Inhalation Toxicity: 90-day study" from the Organization for Economic Co-operation and Development (8). 


\section{MATERIALS AND METHODS}

Animal husbandry and maintenance. Specific-pathogen-free SD rats of each sex (5 weeks old) were purchased from Japan SLC Inc. (Shizuoka, Japan) and acclimated for 1 week. The environmental conditions were controlled to provide a temperature of $22 \pm 3^{\circ} \mathrm{C}$, relative humidity of $50 \pm 20 \%, 12: 12 \mathrm{~h}$ light : dark cycle, and fresh air ventilation (10 15 times per hour). The rats were housed singly in stainless steel wire mesh cages $(W 750 \mathrm{~mm} \times \mathrm{L} 220 \mathrm{~mm} \times$ $\mathrm{H} 180 \mathrm{~mm}$ ) and supplied with commercial rodent diet (Lab$\mathrm{Diet}^{\mathbb{B}}$, St. Louis, MO, USA) and filtered tap water ad libitum. The study was performed at Occupational Safety and Health Research Institute, KOSHA, and was approved by the Animal Care and Use Committee (IACUC-1401).

Test chemical and exposure. 1-Chloropropane $\left(\mathrm{CH}_{3} \mathrm{CH}_{2} \mathrm{CH}_{2} \mathrm{Cl}\right.$, CAS No. 540-54-5) was purchased from Yulchon Noterram Co., Ltd. (Lot N0. 140321, Korea). Whole body exposure chamber (SIS-20, Shibata Co., Japan), including a gas generator (VG-4R, Shibata Co., Japan) were used to expose to 1 -chloropropane. The rats were exposed to $0,310,1250,5000 \mathrm{ppm}$ for $6 \mathrm{~h} /$ day, 5 day/ week for 13 weeks in accordance with the test No. 413 (Subchronic Inhalation Toxicity, 2009) by Organization for Economic Co-operation and Development (OECD) (8). The inhalation exposure was conducted from 10:00 to 16:00 in stainless steel chamber. The experimental design was based on the routine working schedule of workers and the major exposure route for the test substance.

Condition in the inhalation chamber. Temperature, relative humidity, pressure, and air ventilation in the chambers were recorded using an environmental controller (ICS20RG. Shibata Co., Japan). The temperature and relative humidity were maintained at $22.3 \sim 22.9^{\circ} \mathrm{C}$ and $51.2 \sim 56.4 \%$, respectively. The concentrations of 1 -chloropropane in the chambers were calibrated with a standard chemical (RIGAS, Daejeon, Korea). The conditions used for detecting 1 -chloropropane by gas chromatography (GCS-14PFFS, Shimadzu Co., Japan) were as follows: detector; (flame ionization detector); column (silicon DC-200 15\% chromosorb with mesh of $80 / 100$ and a $0.5 \mathrm{~m}$ length); detector temperature, $100^{\circ} \mathrm{C}$; oven temperature, $120^{\circ} \mathrm{C}$; injector temperature, $120^{\circ} \mathrm{C}$; and injection volume, $1 \mathrm{~mL}$ of gas sample. The vapor concentrations of 1 -chloropropane in the chambers were measured every $15 \mathrm{~min}$ during exposure, and were controlled to be within $\pm 5 \%$ of the target concentration using a computer. The mean concentration measured every $30 \mathrm{~min}$ for $6 \mathrm{~h}$ was taken as the value on a given day. This was then averaged over the 13 week exposure period in order to obtain the mean and standard deviations, and the daily gas concentrations in the three chambers were measured at $309.7 \pm 3.35,1250.7 \pm 11.21$, and $5005.2 \pm 25.86$ ppm, respectively.

Experimental groups and selection of concentration. Forty rats of each sex were randomly assigned to the following four groups (10 per sex per group): control group $(0 \mathrm{ppm})$, low dose group (310 ppm), middle dose group $(1,250 \mathrm{ppm})$, and high dose group $(5,000 \mathrm{ppm})$. All the rats were sacrificed after the treatment of 13 weeks. The doses were selected on the basis of acute toxicity study performed previously (data not shown). In this study, the concentration of 310,1,250 and 5,000 ppm were then selected as low dose, middle dose, high dose, using scaling factor $\times 4$ according to Globally Harmonized Systems of Classification and Labeling of Chemicals (GHS).

Clinical signs, body weight and food consumption. All animals were observed daily once for mortality and clinical signs throughout the study. They were weighed individually just before inhalation on day 1 and once per week thereafter. Daily food consumption was measured before the initiation of inhalation and once per week thereafter. Food consumption was evaluated by subtracting leftover feed from the total feed supplied.

Ophthalmological examinations and motor activity measurement. The external eye examinations were conducted prior to the experiment. In the last week of the study, the ocular fundi were examined using a slit lamp (Portable Slit lamp SL-14, Kowa, Japan) and a hand-held fundus camera (Genesis K9L22, Kowa, Japan) after being treated with mydriatic eye drops (Mydrin-P, Santen Pharmaceutical Co, Japan). The motor activities were measured at the end of the exposure period with a motor activity monitoring system (MCMEA-8, Sibata Co., Japan).

Urinalysis. During the last week of exposure, urinalysis was conducted with fresh urine to determine the specific gravity, $\mathrm{pH}$, protein, glucose, ketone body, occult blood, bilirubin, urobilinogen, nitrite, and leukocyte contents by using test kits (BioGen 10 SGLM, Yeongdong Diagnotics Co., Republic of Korea) or a urine chemistry analyzer (Uriscan S-300, Yeongdong Diagnotics Co., Republic of Korea).

Hematology. All animals were fasted overnight prior to necropsy and blood collection. Blood samples were drawn from the abdominal aorta by using a syringe with a 24-gauge needle under Isoflurane anesthesia (Ilsung Pharm, Korea). The blood samples were collected into vacutainers containing EDTA-3K (Beckton Dickinson, USA), and analyzed within 20 min using an automatic hematology analyzer (Hemavet 950, CDC Technology, USA) and were measured using an automated coagulation analyzer (Huma clot duo, Human Germany) with plasma obtained after 
blood was mixed with $3.2 \%$ sodium citrate (9NC sodium citrate, Beckton Dickinson, USA). The following parameters were determined: total erythrocyte counts (RBC), hemoglobin concentration (HGB), hematocrit (HCT), mean cell volume (MCV), mean cell hemoglobin $(\mathrm{MCH})$, mean cell hemoglobin concentration (MCHC), red cell distribution (RDW), platelet count (PLT), mean platelet volume (MPV), prothrombin time (PT) and activated partial thromboplastin time (APTT), whole leukocyte counts (WBC), neutrophils (NEU, \%), eosinophils (EOS, \%), basophils (BASO, \%), lymphocytes (LYM, \%) and monocytes (MONO, \%).

Serum biochemistry. Blood samples were centrifuged at 3,000 rpm for $10 \mathrm{~min}$ within $1 \mathrm{hr}$ after collection. The sera were stored at $-80^{\circ} \mathrm{C}$ in a freezer prior to analysis. The following serum chemistry parameters were evaluated using an autoanalyzer (TBA-20FR, Toshiba, Japan) and an electrolyte analyzer (DRICHEM 800, FUJI, Japan): total protein (TP), albumin (ALB), blood urea nitrogen (BUN), creatinine (CREA), total bilirubin (TBIL), alanine aminotransferase (ALT), aspartate aminotransferase (AST), lactate dehydrogenase (LDH), alkaline phosphatase (ALP), glucose (GLU), total cholesterol (TCHO), triglycerides (TG), $\gamma$-glutamyl transferase ( $\gamma$-GT), CPK (Creatine Phosphokinase), calcium (Ca), inorganic phosphorus (IP), sodium $\left(\mathrm{Na}^{+}\right)$, and potassium $\left(\mathrm{K}^{+}\right)$, and chloride $\left(\mathrm{Cl}^{-}\right)$.

Necropsy, organ weight, and histopathological evaluations. Gross examinations of the organs in the cranial, thoracic, and abdominal cavities of the sacrificed rats were conducted. The absolute and relative weights (organ to body weight) of the brain, thymus, lung, heart, liver, spleen, kidney (left and right), adrenals (left and right), testis (left and right), ovaries (left and right) were measured. The following tissues were removed from each animal at necropsy: liver, kidney, adrenal gland, heart, lung, cerebrum, cerebellum, olfactory bulb, pituitary, spleen, seminal vesicle, prostate, testis, epididymis, ovary, uterus, vagina, tongue, trachea, esophagus, thymus, thyroid, stomach, duodenum, urinary bladder, small/large intestine, eye/harderian gland, skeletal muscle, sciatic nerve, pancreas, mesenteric lymph node, femur, larynx, and nasal cavity. The nasal cavity was sectioned at three levels (Level 1: posterior to the upper incisors, level 2: incisive papilla, level 3: first molar teeth). The eyes were fixed in Davidson's solution and the testis was fixed in Bouin's solution. Other organs were preserved in $10 \%$ neutral buffered formalin. And these organs were embedded in paraffin, sectioned at $3 \sim 4 \mu \mathrm{m}$, stained with hematoxylin and eosin, and examined microscopically with low power field to high power field.

Statistical analysis. The data were expressed as the mean \pm standard deviation. The differences in parameters among the groups were evaluated using SPSS (ver. 19.0, IBM, USA) or SigmaPlot (ver. 13.0, SYSTAT, USA). The homogeneity of variance was determined by Levene's test, which was followed by either one-way analysis of variance (ANOVA) for samples with homogenous variance or the Kruskal-Wallis test for samples with heterogeneous variance. Duncan test (for ANOVA) or Dunnett T3 (for Kruskal-Wallis) was used for multiple comparisons of the control group with each treatment group if the first statistical result was significant.

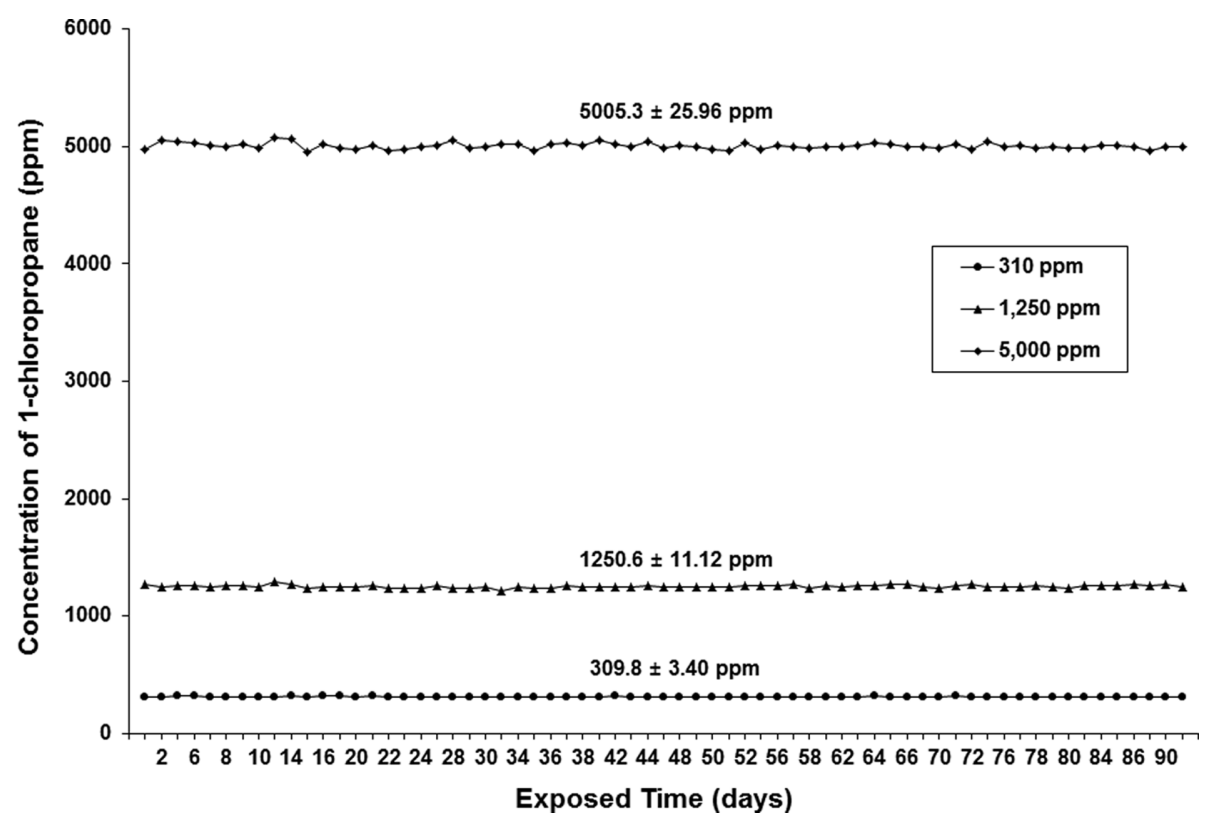

Fig. 1. Changes of concentration in inhalation chamber during the experiment. 


\section{RESULTS}

Chamber monitoring. The average chamber condition were $22.3 \sim 22.9^{\circ} \mathrm{C}, 51.2 \sim 56.3 \%$ relative humidity, $-10.1 \sim 9.7 \mathrm{mmH} 2 \mathrm{O}$ pressure, and $203.6 \sim 213.6 \mathrm{~L} / \mathrm{min}$ flow rate. The average concentration of 1 -chloropropane during the study was as follows: $309.7 \pm 3.35 \mathrm{ppm}$ for low dose, $1,250.7 \pm 11.21 \mathrm{ppm}$ for medium dose, and 5,005.2 $\pm 25.86 \mathrm{ppm}$ for high dose. The daily mean chamber concentration was with $\pm 10 \%$ of the target concentration (Fig. 1).

Clinical signs: No death or adverse clinical signs were observed in any of the 1 -chloropropane groups.

Ophthalmological examination and motor activity measurement: No adverse symptoms were observed in all groups during the study period (data not shown).

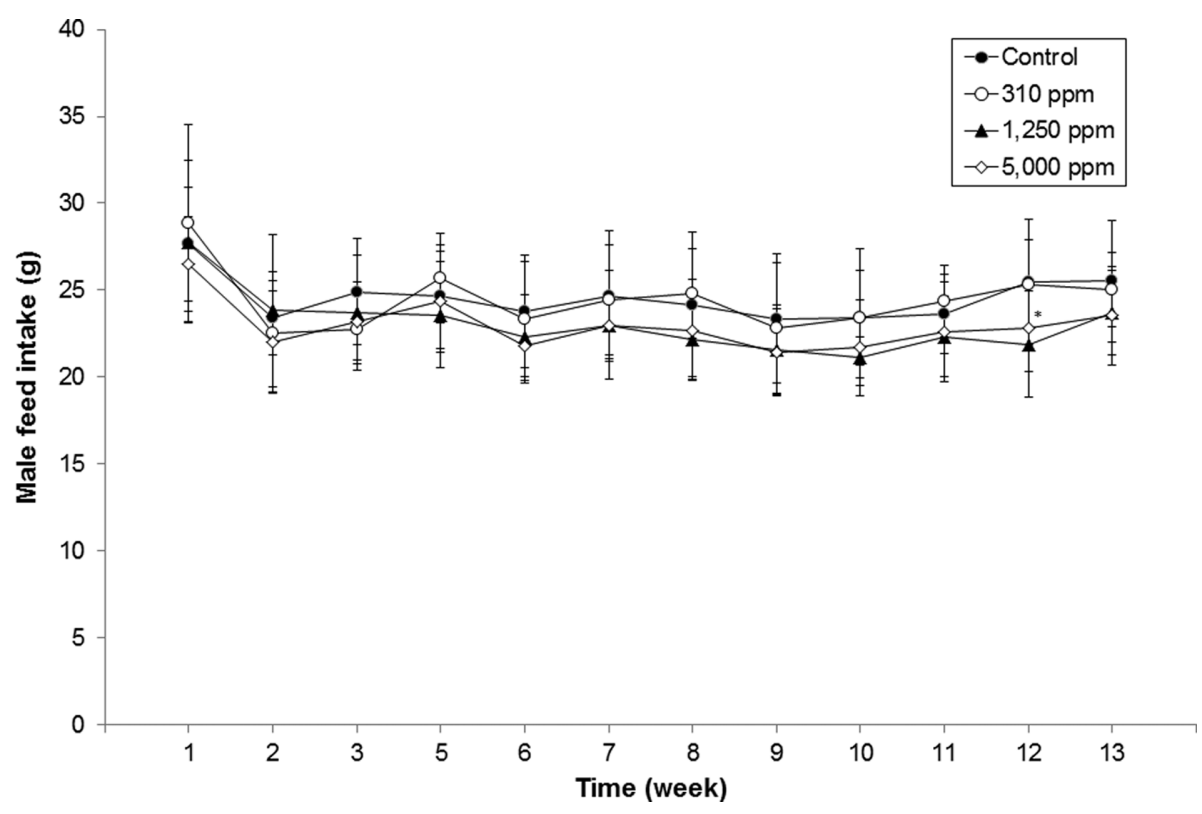

Fig. 2. Daily mean food consumption of male rats exposed to 1-chloropropane for 13 weeks. Significant differences as compared with that of control group: ${ }^{*} p<0.05$.

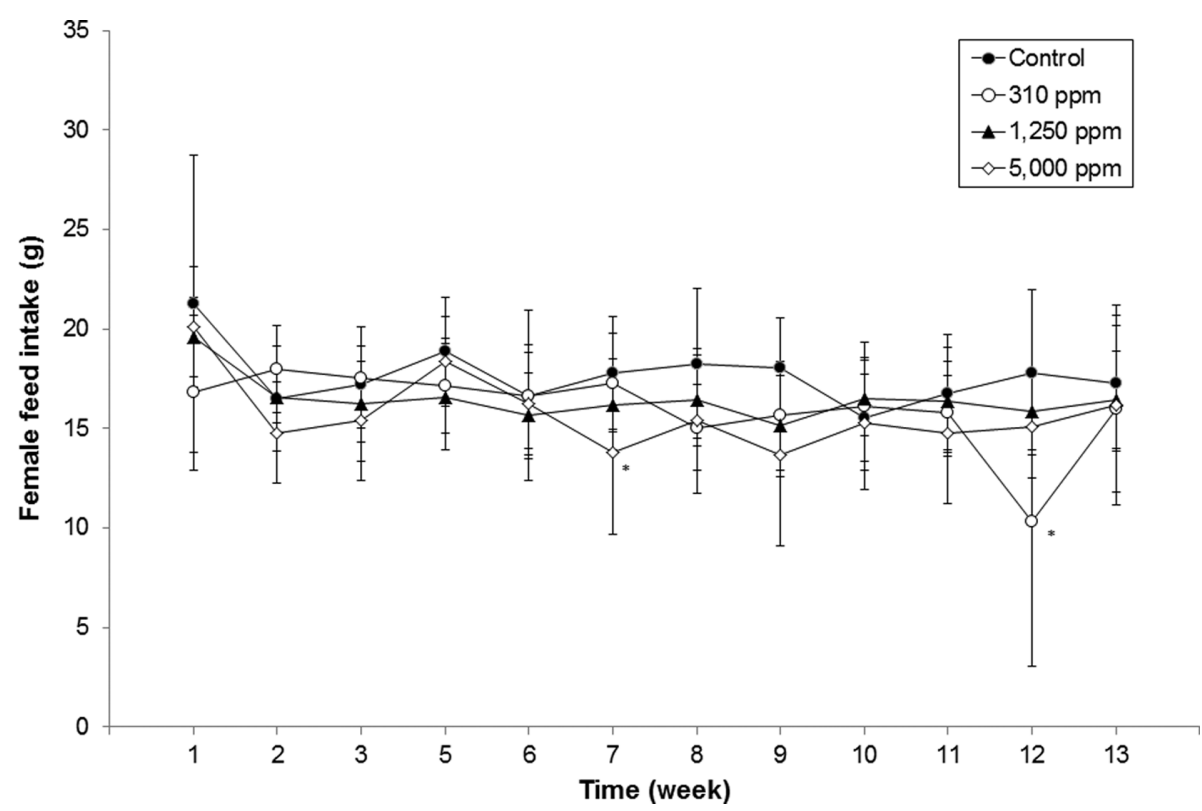

Fig. 3. Daily mean food consumption of female rats exposed to 1-chloropropane for 13 weeks. Significant differences as compared with that of control group: ${ }^{*} p<0.05$. 
Body weight, and food consumption. Body weight did not significantly changed in all male and female animals (data not shown). Food consumption decreased significantly in male rats exposed to $1,250 \mathrm{ppm}$ in week 12 and in female rats exposed to $5,000 \mathrm{ppm}$ in week 7 and to
310 ppm in week $12(p<0.05)$ (Fig. 2 and 3).

Urinalysis. No significant changes were observed in all 1-chloropropane groups during the study period (data not shown).

Table 1. Serum biochemical values of male rats exposed to 1-chloropropane for 13 weeks

\begin{tabular}{|c|c|c|c|c|c|}
\hline \multirow{2}{*}{ Tests } & \multirow{2}{*}{ Units } & \multicolumn{4}{|c|}{ Groups (ppm) } \\
\hline & & G1 (0) & G2 (310) & G3 $(1,250)$ & G4 $(5,000)$ \\
\hline ТP & $\mathrm{g} / \mathrm{dL}$ & $7.37 \pm 0.31$ & $7.48 \pm 0.34$ & $7.45 \pm 0.43$ & $7.64 \pm 0.27$ \\
\hline ALB & $\mathrm{g} / \mathrm{dL}$ & $4.33 \pm 0.17$ & $4.44 \pm 0.13$ & $4.37 \pm 0.21$ & $4.52 \pm 0.17$ \\
\hline BUN & $\mathrm{mg} / \mathrm{dL}$ & $17.26 \pm 2.02$ & $17.05 \pm 2.31$ & $17.98 \pm 2.64$ & $19.12 \pm 2.20$ \\
\hline CREA & $\mathrm{mg} / \mathrm{dL}$ & $0.54 \pm 0.05$ & $0.52 \pm 0.04$ & $0.55 \pm 0.05$ & $0.55 \pm 0.05$ \\
\hline TBIL & $\mathrm{mg} / \mathrm{dL}$ & $0.19 \pm 0.02$ & $0.19 \pm 0.02$ & $0.22 \pm 0.02^{* *}$ & $0.20 \pm 0.01^{* *}$ \\
\hline ALT & $\mathrm{IU} / \mathrm{L}$ & $74.6 \pm 38.74$ & $44.3 \pm 9.82^{*}$ & $71.8 \pm 54.36$ & $63.6 \pm 31.28$ \\
\hline AST & IU/L & $150.5 \pm 80.03$ & $97.3 \pm 19.62$ & $123.5 \pm 64.36$ & $118.4 \pm 66.81$ \\
\hline LDH & IU/L & $940.1 \pm 659.99$ & $916.0 \pm 443.58$ & $690.7 \pm 386.40$ & $642.6 \pm 494.35$ \\
\hline ALP & IU/L & $408.0 \pm 64.46$ & $404.6 \pm 131.87$ & $412.4 \pm 98.46$ & $422.6 \pm 163.03$ \\
\hline GLU & $\mathrm{mg} / \mathrm{dL}$ & $246.5 \pm 25.01$ & $235.8 \pm 22.84$ & $219.0 \pm 10.11^{* *}$ & $196.5 \pm 17.45^{* *}$ \\
\hline TCHO & $\mathrm{mg} / \mathrm{dL}$ & $84.5 \pm 13.48$ & $89.1 \pm 12.74$ & $76.7 \pm 11.98$ & $95.8 \pm 19.38$ \\
\hline TG & $\mathrm{mg} / \mathrm{dL}$ & $53.2 \pm 19.94$ & $55.4 \pm 21.63$ & $46.2 \pm 16.40$ & $66.0 \pm 23.77$ \\
\hline$\gamma$-GT & $\mathrm{IU} / \mathrm{L}$ & $299.8 \pm 232.08$ & $271.8 \pm 115.40$ & $225.3 \pm 85.79^{* *}$ & $194.6 \pm 124.27^{* *}$ \\
\hline CPK & IU/L & $11.05 \pm 0.28$ & $11.01 \pm 0.29$ & $11.12 \pm 0.53$ & $11.12 \pm 0.26$ \\
\hline $\mathrm{Ca}^{2+}$ & $\mathrm{IU} / \mathrm{L}$ & $6.50 \pm 0.59$ & $6.96 \pm 1.14$ & $6.17 \pm 1.84$ & $6.49 \pm 0.73$ \\
\hline IP & $\mathrm{mg} / \mathrm{dL}$ & $0.37 \pm 0.50$ & $0.31 \pm 0.16$ & $0.84 \pm 0.51$ & $0.82 \pm 0.60$ \\
\hline $\mathrm{Na}^{+}$ & $\mathrm{mmol} / \mathrm{L}$ & $140.0 \pm 1.15$ & $140.3 \pm 0.95$ & $140.4 \pm 1.26$ & $139.8 \pm 0.92$ \\
\hline $\mathrm{K}^{+}$ & $\mathrm{mmol} / \mathrm{L}$ & $5.06 \pm 0.40$ & $4.97 \pm 0.31$ & $5.05 \pm 0.49$ & $5.14 \pm 0.55$ \\
\hline $\mathrm{Cl}^{-}$ & $\mathrm{mmol} / \mathrm{L}$ & $103.8 \pm 1.40$ & $103.6 \pm 1.17$ & $102.9 \pm 1.20$ & $102.4 \pm 0.97^{*}$ \\
\hline
\end{tabular}

All values are expressed as mean \pm SD.

Significant differences as compared with controlgroup: ${ }^{*} p<0.05,{ }^{* *} p<0.01$.

Table 2. Serum biochemical values of female rats exposed to 1-chloropropane for 13 weeks

\begin{tabular}{|c|c|c|c|c|c|}
\hline \multirow{2}{*}{ Tests } & \multirow{2}{*}{ Units } & \multicolumn{4}{|c|}{ Groups (ppm) } \\
\hline & & G1 (0) & G2 (310) & G3 $(1,250)$ & G4 $(5,000)$ \\
\hline $\mathrm{TP}$ & $\mathrm{g} / \mathrm{dL}$ & $6.95 \pm 0.33$ & $6.87 \pm 0.23$ & $7.17 \pm 0.35$ & $7.51 \pm 0.57^{* *}$ \\
\hline ALB & $\mathrm{g} / \mathrm{dL}$ & $4.29 \pm 0.22$ & $4.24 \pm 0.20$ & $4.49 \pm 0.21$ & $4.64 \pm 0.39^{* * *}$ \\
\hline BUN & $\mathrm{mg} / \mathrm{dL}$ & $16.01 \pm 1.89$ & $16.41 \pm 1.85$ & $15.66 \pm 1.51$ & $16.15 \pm 1.25$ \\
\hline CREA & $\mathrm{mg} / \mathrm{dL}$ & $0.46 \pm 0.05$ & $0.48 \pm 0.07$ & $0.47 \pm 0.05$ & $0.49 \pm 0.03$ \\
\hline TBIL & $\mathrm{mg} / \mathrm{dL}$ & $0.11 \pm 0.03$ & $0.12 \pm 0.02$ & $0.11 \pm 0.01$ & $0.13 \pm 0.03$ \\
\hline ALT & IU/L & $171.9 \pm 240.90$ & $75.6 \pm 22.51$ & $76.3 \pm 28.67$ & $82.3 \pm 53.73$ \\
\hline AST & $\mathrm{IU} / \mathrm{L}$ & $223.0 \pm 198.93$ & $145.7 \pm 57.70$ & $148.6 \pm 57.60$ & $182.2 \pm 88.98$ \\
\hline LDH & $\mathrm{IU} / \mathrm{L}$ & $862.6 \pm 462.24$ & $948.7 \pm 535.12$ & $720.3 \pm 427.83$ & $707.4 \pm 467.83$ \\
\hline ALP & $\mathrm{IU} / \mathrm{L}$ & $268.6 \pm 131.76$ & $205.6 \pm 91.06$ & $244.9 \pm 147.80$ & $316.2 \pm 244.66$ \\
\hline GLU & $\mathrm{mg} / \mathrm{dL}$ & $178.0 \pm 21.34$ & $181.1 \pm 20.90$ & $183.6 \pm 18.82$ & $175.1 \pm 22.74$ \\
\hline TCHO & $\mathrm{mg} / \mathrm{dL}$ & $103.8 \pm 15.63$ & $101.4 \pm 14.37$ & $103.0 \pm 12.22$ & $115.7 \pm 22.43$ \\
\hline TG & $\mathrm{mg} / \mathrm{dL}$ & $25.7 \pm 7.66$ & $27.3 \pm 15.98$ & $27.7 \pm 6.02$ & $32.3 \pm 15.90$ \\
\hline$\gamma$-GT & $\mathrm{IU} / \mathrm{L}$ & $296.4 \pm 166.82$ & $300.6 \pm 151.02$ & $223.4 \pm 112.02$ & $225.0 \pm 140.67$ \\
\hline CPK & $\mathrm{IU} / \mathrm{L}$ & $10.39 \pm 0.23$ & $10.44 \pm 0.26$ & $10.38 \pm 0.33$ & $10.64 \pm 0.51$ \\
\hline $\mathrm{Ca}^{2+}$ & $\mathrm{IU} / \mathrm{L}$ & $5.80 \pm 0.24$ & $5.80 \pm 0.35$ & $5.70 \pm 0.26$ & $5.81 \pm 0.32$ \\
\hline IP & $\mathrm{mg} / \mathrm{dL}$ & $1.77 \pm 2.68$ & $0.53 \pm 0.57$ & $0.37 \pm 0.23$ & $0.41 \pm 0.31$ \\
\hline $\mathrm{Na}^{+}$ & $\mathrm{mmol} / \mathrm{L}$ & $138.7 \pm 1.16$ & $138.9 \pm 0.93$ & $138.2 \pm 1.14$ & $138.4 \pm 0.97$ \\
\hline $\mathrm{K}^{+}$ & $\mathrm{mmol} / \mathrm{L}$ & $4.73 \pm 0.17$ & $4.81 \pm 0.21$ & $4.85 \pm 0.24$ & $4.70 \pm 0.37$ \\
\hline $\mathrm{Cl}^{-}$ & $\mathrm{mmol} / \mathrm{L}$ & $103.8 \pm 4.10$ & $104.2 \pm 1.64$ & $103.1 \pm 2.08$ & $102.6 \pm 3.41$ \\
\hline
\end{tabular}

All values are expressed as mean \pm SD.

Significant differences as compared with control group: ${ }^{*} p<0.05,{ }^{* *} p<0.01$. 
Hematology. No statistically significant hematological changes in comparison with the control group were observed in any of all 1 -chloropropane tested groups.

Serum biochemistry. Total bilirubin was significantly increased in males of 1,250 and 5,000 ppm groups $(p<$ 0.01 ). Glucose and $\gamma$-GT were significantly decreased in males of 1,250 and 5,000 ppm groups compared to those of control group $(p<0.01)$. ALT and $\mathrm{Cl}^{-}$were significantly decreased in male 310 and 5,000 ppm group, respectively $(p<0.05)$ (Table 1). Total protein and albumin was significantly increased in females of 5,000 ppm group in comparison with those of the control group $(p<0.01)$ (Table 2$)$.

Organ weights. No statistically significant absolute organ weight changes were observed in any of the male groups in comparison with the control group (Table 3).
However, the absolute weights of left ovary and spleen were significantly increased in $310 \mathrm{ppm}$ group $(p<0.05$ or $p<0.01)$ and the absolute weights of right kidney and liver were significantly increased in $5,000 \mathrm{ppm}$ group compared to those of the control group ( $p<0.05$ or $p<0.01$ ) (Table $4)$. The relative organ weights of right kidney and liver were significantly increased in males of 5,000 ppm group compared to those of the control group $(p<0.05$ or $p<$ 0.01 ) (Table 5). The relative weights of left kidney and liver were significantly increased in females of 5,000 ppm group compared to those of the control group $(p<0.05$ or $p<$ 0.01) (Table 6).

Gross and histopathological evaluations. No gross lesions were observed in all groups. Vacuolation of acinar cells were noted in the pancreas: in males; minimal grade in $3 / 10,2 / 10,2 / 10$, and $1 / 10$, slight grade in $0 / 10,4 / 10,2 / 10$,

Table 3. Absolute organ weights of male rats exposed to 1-chloropropane for 13 weeks

\begin{tabular}{|c|c|c|c|c|}
\hline \multirow{2}{*}{ Organ weights (g) } & \multicolumn{4}{|c|}{ Groups (ppm) } \\
\hline & G1 (0) & $\mathrm{G} 2(310)$ & G3 $(1,250)$ & G4 $(5,000)$ \\
\hline Thymus & $0.368 \pm 0.094$ & $0.371 \pm 0.086$ & $0.369 \pm 0.066$ & $0.349 \pm 0.047$ \\
\hline Heart & $1.270 \pm 0.168$ & $1.284 \pm 0.148$ & $1.254 \pm 0.122$ & $1.281 \pm 0.117$ \\
\hline Adrenal gland L & $0.035 \pm 0.004$ & $0.036 \pm 0.005$ & $0.036 \pm 0.005$ & $0.039 \pm 0.007$ \\
\hline Adrenal gland $\mathrm{R}$ & $0.034 \pm 0.005$ & $0.038 \pm 0.006$ & $0.038 \pm 0.007$ & $0.036 \pm 0.006$ \\
\hline Testis L & $1.758 \pm 0.127$ & $1.844 \pm 0.135$ & $1.772 \pm 0.147$ & $1.845 \pm 0.082$ \\
\hline Testis R & $1.800 \pm 0.180$ & $1.954 \pm 0.247$ & $1.823 \pm 0.146$ & $1.879 \pm 0.046$ \\
\hline Lung L & $0.450 \pm 0.063$ & $0.504 \pm 0.114$ & $0.445 \pm 0.058$ & $0.451 \pm 0.050$ \\
\hline Lung R & $0.821 \pm 0.119$ & $0.887 \pm 0.085$ & $0.817 \pm 0.119$ & $0.839 \pm 0.129$ \\
\hline Kidney L & $1.387 \pm 0.177$ & $1.451 \pm 0.219$ & $1.423 \pm 0.098$ & $1.485 \pm 0.136$ \\
\hline Kidney R & $1.376 \pm 0.193$ & $1.451 \pm 0.208$ & $1.424 \pm 0.117$ & $1.530 \pm 0.164$ \\
\hline Spleen & $0.705 \pm 0.153$ & $0.659 \pm 0.124$ & $0.672 \pm 0.089$ & $0.726 \pm 0.092$ \\
\hline Liver & $12.298 \pm 1.403$ & $12.234 \pm 1.404$ & $12.507 \pm 1.118$ & $13.478 \pm 1.494$ \\
\hline Brain & $2.199 \pm 0.328$ & $2.188 \pm 0.063$ & $2.117 \pm 0.057$ & $2.159 \pm 0.083$ \\
\hline
\end{tabular}

Table 4. Absolute organ weights of female rats exposed to 1-chloropropane for 13 weeks

\begin{tabular}{lcccc}
\hline \hline \multirow{2}{*}{ Organ weights $(\mathrm{g})$} & \multicolumn{3}{c}{ Groups $(\mathrm{ppm})$} \\
\cline { 2 - 5 } & $\mathrm{G} 1(0)$ & $\mathrm{G} 2(310)$ & $\mathrm{G} 3(1,250)$ & $\mathrm{G} 4(5,000)$ \\
\hline Thymus & $0.281 \pm 0.035$ & $0.292 \pm 0.053$ & $0.284 \pm 0.046$ & $0.285 \pm 0.041$ \\
Heart & $0.855 \pm 0.057$ & $0.907 \pm 0.086$ & $0.914 \pm 0.076$ & $0.909 \pm 0.044$ \\
Adrenal gland L & $0.044 \pm 0.007$ & $0.063 \pm 0.075$ & $0.044 \pm 0.010$ & $0.044 \pm 0.007$ \\
Adrenal gland R & $0.041 \pm 0.009$ & $0.046 \pm 0.023$ & $0.037 \pm 0.010$ & $0.043 \pm 0.009$ \\
Ovary L & $0.082 \pm 0.011$ & $0.067 \pm 0.022^{*}$ & $0.092 \pm 0.027$ & $0.083 \pm 0.012$ \\
Ovary R & $0.089 \pm 0.035$ & $0.087 \pm 0.021$ & $0.089 \pm 0.014$ & $0.095 \pm 0.012$ \\
Lung L & $0.380 \pm 0.023$ & $0.354 \pm 0.019$ & $0.365 \pm 0.025$ & $0.375 \pm 0.022$ \\
Lung R & $0.671 \pm 0.067$ & $0.637 \pm 0.033$ & $0.663 \pm 0.158$ & $0.676 \pm 0.047$ \\
Kidney L & $0.876 \pm 0.063$ & $0.875 \pm 0.054$ & $0.906 \pm 0.039$ & $0.959 \pm 0.076$ \\
Kidney R & $0.901 \pm 0.055$ & $0.889 \pm 0.069$ & $0.905 \pm 0.079$ & $0.962 \pm 0.077^{* *}$ \\
Spleen & $0.465 \pm 0.036$ & $0.462 \pm 0.038^{* *}$ & $0.478 \pm 0.082$ & $0.494 \pm 0.064$ \\
Liver & $6.683 \pm 0.624$ & $6.492 \pm 0.561$ & $6.950 \pm 0.722$ & $7.545 \pm 0.825^{*}$ \\
Brain & $1.987 \pm 0.101$ & $2.044 \pm 0.052$ & $2.056 \pm 0.103$ & $2.061 \pm 0.094$ \\
\hline
\end{tabular}

*All values are expressed as mean \pm SD.

Significant differences as compared with that of control group : ${ }^{*} p<0.05,{ }^{* *} p<0.01$. 
Table 5. Relative organ weights of male rats exposed to 1-chloropropane for 13 weeks

\begin{tabular}{lcccc}
\hline \hline \multirow{2}{*}{ Organ weights (\%) } & \multicolumn{3}{c}{ Groups (ppm) } \\
\cline { 2 - 5 } & G1 $(0)$ & G2 (310) & G3 $(1,250)$ & G4 (5,000) \\
\hline Thymus & $0.082 \pm 0.021$ & $0.080 \pm 0.016$ & $0.083 \pm 0.014$ & $0.078 \pm 0.010$ \\
Heart & $0.282 \pm 0.017$ & $0.278 \pm 0.017$ & $0.282 \pm 0.018$ & $0.288 \pm 0.037$ \\
Adrenal gland L & $0.008 \pm 0.001$ & $0.008 \pm 0.001$ & $0.008 \pm 0.001$ & $0.009 \pm 0.001$ \\
Adrenal gland R & $0.007 \pm 0.001$ & $0.008 \pm 0.001$ & $0.008 \pm 0.001$ & $0.008 \pm 0.001$ \\
Testis L & $0.395 \pm 0.044$ & $0.401 \pm 0.023$ & $0.400 \pm 0.035$ & $0.417 \pm 0.056$ \\
Testis R & $0.402 \pm 0.033$ & $0.424 \pm 0.041$ & $0.411 \pm 0.034$ & $0.424 \pm 0.057$ \\
Lung L & $0.100 \pm 0.007$ & $0.109 \pm 0.019$ & $0.100 \pm 0.008$ & $0.101 \pm 0.008$ \\
Lung R & $0.183 \pm 0.016$ & $0.193 \pm 0.022$ & $0.183 \pm 0.018$ & $0.188 \pm 0.028$ \\
Kidney L & $0.308 \pm 0.014$ & $0.314 \pm 0.030$ & $0.322 \pm 0.032$ & $0.332 \pm 0.026$ \\
Kidney R & $0.306 \pm 0.021$ & $0.314 \pm 0.027$ & $0.322 \pm 0.032$ & $0.342 \pm 0.025^{*}$ \\
Spleen & $0.156 \pm 0.022$ & $0.144 \pm 0.028$ & $0.151 \pm 0.014$ & $0.162 \pm 0.016$ \\
Liver & $2.739 \pm 0.122$ & $2.648 \pm 0.166$ & $2.817 \pm 0.183$ & $3.007 \pm 0.115^{* *}$ \\
Brain & $0.493 \pm 0.078$ & $0.477 \pm 0.041$ & $0.479 \pm 0.036$ & $0.485 \pm 0.044$ \\
\hline
\end{tabular}

*All values are expressed as mean \pm SD.

Significant differences as compared with that of control group: ${ }^{*} p<0.05,{ }^{* *} p<0.01$.

Table 6. Relative organ weights of female rats exposed to 1-chloropropane for 13 weeks

\begin{tabular}{lcccc}
\hline \hline \multirow{2}{*}{ Organ weights (\%) } & \multicolumn{3}{c}{ Groups (ppm) } \\
\cline { 2 - 5 } & G1 $(0)$ & G2 (310) & G3 (1,250) & G4 (5,000) \\
\hline Thymus & $0.108 \pm 0.018$ & $0.111 \pm 0.015$ & $0.110 \pm 0.015$ & $0.109 \pm 0.012$ \\
Heart & $0.328 \pm 0.019$ & $0.346 \pm 0.041$ & $0.354 \pm 0.030$ & $0.349 \pm 0.017$ \\
Adrenal gland L & $0.017 \pm 0.003$ & $0.023 \pm 0.026$ & $0.017 \pm 0.004$ & $0.017 \pm 0.003$ \\
Adrenal gland R & $0.016 \pm 0.003$ & $0.017 \pm 0.008$ & $0.015 \pm 0.004$ & $0.016 \pm 0.003$ \\
Testis L & $0.031 \pm 0.005$ & $0.026 \pm 0.008$ & $0.035 \pm 0.009$ & $0.032 \pm 0.004$ \\
Testis R & $0.034 \pm 0.014$ & $0.033 \pm 0.008$ & $0.035 \pm 0.006$ & $0.036 \pm 0.004$ \\
Lung L & $0.146 \pm 0.009$ & $0.135 \pm 0.009$ & $0.142 \pm 0.011$ & $0.144 \pm 0.007$ \\
Lung R & $0.258 \pm 0.029$ & $0.243 \pm 0.019$ & $0.255 \pm 0.048$ & $0.260 \pm 0.019$ \\
Kidney L & $0.337 \pm 0.029$ & $0.333 \pm 0.015$ & $0.352 \pm 0.030$ & $0.368 \pm 0.021^{*}$ \\
Kidney R & $0.346 \pm 0.030$ & $0.338 \pm 0.014$ & $0.351 \pm 0.030$ & $0.369 \pm 0.027$ \\
Spleen & $0.179 \pm 0.018$ & $0.176 \pm 0.016$ & $0.185 \pm 0.027$ & $0.190 \pm 0.024$ \\
Liver & $2.567 \pm 0.250$ & $2.474 \pm 0.214$ & $2.683 \pm 0.091$ & $2.891 \pm 0.256^{* *}$ \\
Brain & $0.764 \pm 0.059$ & $0.781 \pm 0.067$ & $0.799 \pm 0.069$ & $0.791 \pm 0.041$ \\
\hline
\end{tabular}

All values are expressed as mean $\pm S D$.

Significant differences as compared with that of control group: ${ }^{*} p<0.05$, ${ }^{* *} p<0.01$.

Table 7. Histopathological findings of male rats exposed to 1-chloropropane for 13 weeks

\begin{tabular}{llcccc}
\hline \hline & & \multicolumn{3}{c}{ Group } \\
\cline { 3 - 6 } & & G1 $(0 \mathrm{ppm})$ & $\mathrm{G} 2(310 \mathrm{ppm})$ & $\mathrm{G} 3(1,250 \mathrm{ppm})$ & $\mathrm{G} 4(5,000 \mathrm{ppm})$ \\
\hline \multirow{5}{*}{ Pancreas } & No abnormalities detected & $3 / 10$ & $0 / 10$ & $0 / 10$ & $0 / 10$ \\
& Vacuolation, acinar cells & & & & \\
& Minimal & $7 / 10$ & $2 / 10$ & $5 / 10$ & $0 / 10$ \\
& Slight & $0 / 10$ & $5 / 10$ & $6 / 10$ & $5 / 10$ \\
& Moderate & $0 / 10$ & $3 / 10$ & $3 / 10$ & $5 / 10$ \\
\hline
\end{tabular}

and $4 / 10$, moderate grade in $0 / 10,1 / 10,4 / 10$, and $5 / 10$ of the control group $310,1,250$, and $5,000 \mathrm{ppm}$ group, in females; minimal grade in $7 / 10,2 / 10,1 / 10$, and $0 / 10$, slight grade in $0 / 10,5 / 10,6 / 10$, and $5 / 10$, moderate grade in $0 / 10$, $3 / 10,3 / 10$, and $5 / 10$ of the control group, $310,1,250$, and
5,000 ppm groups (Table 6 and 7) (Fig. 3). The other histopathological findings observed in both sex of all 1 -chloropropane tested groups were also found in the control group or were determined to be accidental changes not accompanied by any dose-response relationship. 
Table 8. Histopathological findings of female rats exposed to 1-chloropropane for 13 weeks

\begin{tabular}{llccrr}
\hline \hline & & \multicolumn{3}{c}{ Group } \\
\cline { 3 - 6 } & & G1 $(0 \mathrm{ppm})$ & $\mathrm{G} 2(310 \mathrm{ppm})$ & $\mathrm{G} 3(1,250 \mathrm{ppm})$ & $\mathrm{G} 4(5,000 \mathrm{ppm})$ \\
\hline \multirow{5}{*}{ Pancreas } & $7 / 10$ & $4 / 10$ & $2 / 10$ & $0 / 10$ \\
& No abnormalities detected & & & \\
& Vacuolation, acinar cells & $3 / 10$ & $2 / 10$ & $2 / 10$ & $1 / 10$ \\
& Minimal & $0 / 10$ & $4 / 10$ & $2 / 10$ & $4 / 10$ \\
& Slight & $0 / 10$ & $0 / 10$ & $4 / 10$ & $5 / 10$ \\
\hline
\end{tabular}
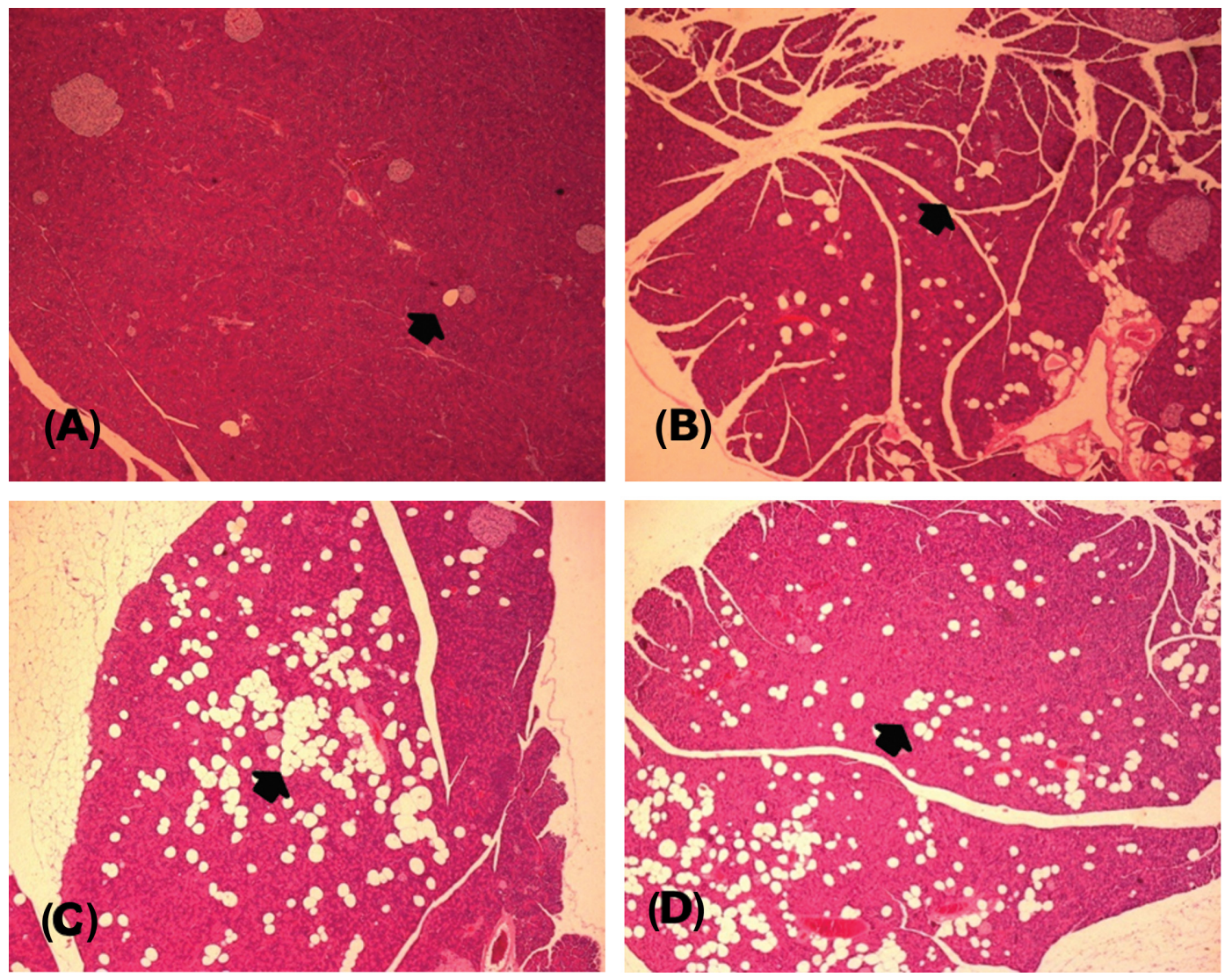

Fig. 4. Histopathology of the pancreas in rats exposed to 1-chloropropane. (A) Control group; (B) low dose group; (C) middle dose group; (D) high dose group. An arrow indicates the vacuolation of acinar cells, H\&E $\times 25$.

\section{DISCUSSION}

This study was performed to evaluate the potential subchronic toxicity of 1 -chloropropane by 13 week repeated inhalation exposure to SD rats at the dose of $0,310,1,250$, and 5,000 ppm. The result revealed that whole body inhalation exposure of 1 -chloropropane caused some adverse effects in serum biochemistry and histopathology.

In food consumption, statistically significant changes in food intake were observed in male rats exposed to 1,250 ppm in week 12 and in female rats exposed to 5,000 ppm in week 7 and to $310 \mathrm{ppm}$ in week 12 . However, these were not considered to be an adverse effect because the change was not dose-dependent and recovered indicating these changes were temporary.

In serum biochemistry, decrease of glucose was observed in males exposed to $1,250 \mathrm{ppm}$ or $5,000 \mathrm{ppm}$, compared to that of control groups. This change could be caused by vacuolation of pancreas because pancreatic acinar cells become degranulated and atrophic with it (9) and that could result in decreased digestion of carbohydrate, precursor of glucose. TP and ALB were increased in females exposed to 5,000 ppm. These changes were potentially considered to be adverse effect because these changes were correlated with the organ weights in liver or kidney. Furthermore, the previous study indicated adverse effect in liver (6). However, the 
reason of sex difference is exactly unknown in this study. Also, $\gamma$-GT and TBIL were observed in males exposed to $1,250 \mathrm{ppm}$ or $5,000 \mathrm{ppm}$, compared to that of control groups. However, these were not regarded as adverse effect because these changes were not dose dependent and were not correlated with the morphological changes in liver. ALT and $\mathrm{Cl}^{-}$were decreased in males exposed to $310 \mathrm{ppm}$ or $5,000 \mathrm{ppm}$, respectively. These changes were not considered to be adverse effect because these changes were not observed in other sex groups and were not correlated with other parameters in serum biochemistry and morphological alterations in liver or kidney.

Organ weights analyses showed that there were statistically significant increase in the absolute and relative organ weight of kidney and liver in males and females of 5,000 ppm group and in the absolute weights of left ovary and spleen in females of $310 \mathrm{ppm}$ group. The change in the absolute and relative organ weight of kidney and liver in females of 5,000 ppm group was considered to be adverse effect because these changes were correlated with the change of TP and ALB. However, other changes were not considered to be adverse effects because they were small in extent, not dose-dependent and associated with morphological alterations in histopathological examination.

In histopathological examination, vacuolation of acinar cells was observed in pancreas of all groups exposed to 1 chloropropane. The result showed the incidence and severity was correlated with the increasing dose of 1 -chloropropane in this study. Also, vacuolation of acinar cells in pancreas has been reported following treatment with numerous agents including arginine, (10), supraphysiologic stimulation with cerulein, a CCK receptor agonist, cyclosporine A (11), choline-deficient, ethionine-supplemented diet (12), palmitoylpentachlorophenol (PPCP) (13), puromycin (14), and 2,3,7,8-tetrachlorodibenzo- $p$-dioxin (TCDD) (15). Therefore, these results indicated the pancreatic vacuolation was induced by the test substance and considered to be the target organ in this study. Apart from the histopathological lesion described above, other histopathological findings were sporadic, not dose-dependent, and attributable to spontaneous lesions in SD rats of the age used in the study (16).

In conclusion, 13-week repeated whole-body inhalation exposure of rats to 1 -chloropropane results in decreased glucose in serum biochemistry and vacuolation in pancreas. And the target organ was determined to be the pancreas. The no observable adverse effect level (NOAEL) was considered to be below $310 \mathrm{ppm} / 6 \mathrm{~h} /$ day, 5 day/week for rats. The present study is expected to provide useful information on the toxic effect of long term inhalation of 1 -chloropropane.

\section{ACKNOWLEDGMENTS}

This work was supported by the Korea Occupational
Safety \& Health Agency, Ministry of Labor, Republic of Korea, and a Grant-in-Aid for chemical hazard assessment, 2014.

\section{REFERENCES}

1. U.S. National Library of Medicine. Hazadrous Substances Data bank. Substance name: 1-chloropropane. Available from: http://toxnet.nlm.nih.gov/cgi-bin/sis/search2/r?dbs+hsdb:@term+ @ DOCNO+5681.

2. Clayton, G.D. and Clayton, F.E. (1981) Party's industrial hygiene and toxicology: volume 2B: Toxicology (3rd edition), John Wiley \& Sons, New York, pp. 3525.

3. Environmental statistics yearbook. (2007) Ministry of Environment. Available from: http://library.me.go.kr/search/DetailView.Popup.ax?sid=11\&cid=180267.

4. MSDS of 1-chloropropance. Available from: http://www.fishersci.ca/viewmsds.do?catNo=AC109951000.

5. Saito-Suzuki, R., Teramoto, S. and Shirasu, Y. (1982) Dominant lethal studies in rats with 1,2-dibromo-3-chloropropane and its structurally related compounds. Mutat. Res., 101, 321327.

6. Bingham, E., Cohrssen, B. and Powell, C.H. (2001) Patty's toxicology volumes 1-9 (5th edition), John Wiley \& Sons, New York, pp. 154.

7. Grant, W.M. (1986) Toxicology of the eye (3rd edition), Charles C. Thomas Publisher, Springfield, pp. 1024.

8. Organisation for Economic Co-operation and Development (OECD). (2009) OECD guidelines for the testing of chemicals 413, Subchronic Inhalation Toxicity (90-day study). Available from: http://www.oecd-ilibrary.org/docserver/download/9741301e.pdf?expires $=1437622438 \&$ id=id\&accname $=$ guest\&checksum=50B62ACC348A8455A23D07CDB33040A0.

9. Gopinath, C., Prentice, D. and Lewis, D.J. (1987) Alimentary system and pancreas in Atlas of experimental toxicological pathology. Vol. 13. Current histopathology, MTP Press Ltd, Lancaster, pp. 61-76.

10. Tani, S., Itoh, H., Okabayashi, Y., Nakamura, T., Fujii, M., Fujisawa, T., Koide, M. and Otsuki, M. (1990) New model of acute necrotizing pancreatitis induced by excessivedoses of arginine in rats. Dig. Dis. Sci., 35, 367-374.

11. Hirano, T., Manabe, T., Ando, K. and Tobe, T. (1992) Acute cytotoxic effect of cyclosporin A on pancreatic acinar cells in rats: protective effect of the synthetic protease inhibitor E3123. Scand. J. Gastroenterol., 27, 103-107.

12. Mareninova, O.A., Hermann, K., French, S.W., O'Konski, M.S., Pandol, S.J., Webster, P., Erickson, A.H., Katunuma, N., Gorelick, F.S., Gukovsky, I. and Gukovskaya, A.S. (2009) Impaired autophagic flux mediates acinar cell vacuole formation and trypsinogen activation in rodent models of acute pancreatitis. J. Clin. Invest., 119, 3340-3355.

13. Ansari, G.A., Kaphalia, B.S. and Boor, P.J. (1987) Selective pancreatic toxicity of palmitoy lpentachorophenol. Toxicology, 46, 57- 63.

14. Longnecker, D.S., Crawford, B.G. and Nadler, D.J. (1975) Recovery of pancreas from mild puromycin-induced injury. A histologic and ultrastructural study in rats. Arch. Pathol., 99, 5-10. 
15. Nyska, A., Jokinen, M.P., Brix, A.E., Sells, D.M., Wyde, M.E., Orzech, D., Haseman, J.K., Flake, G. and Walker, N.J. (2004) Exocrine pancreatic pathology in female Harlan Sprague Dawley rats after chronic treatment with 2,3,7,8-tetrachlorodibenzo- $p$-dioxin and dioxin-like compounds. Envi- ron. Health Perspect., 112, 903-909.

16. Mclnnes, E.F. (2012) Wistar and sprague-dawely rat in Background Lesion of Laboratory Animals. Saunders, Elsevier, New York, pp. 17-36. 